\title{
On the colors of barlenses and their link to B/P bulges
}

\author{
Martín Herrera Endoqui ${ }^{1}$, Eija Laurikainen ${ }^{1}$, Heikki Salo ${ }^{1}$ and \\ Johan H. Knapen ${ }^{2,3}$ \\ ${ }^{1}$ Astronomy research group, University of Oulu, FI-90014, Finland \\ email: martin.herreraendoqui@oulu.fi \\ ${ }^{2}$ Instituto de Astrofísica de Canarias, E-38205 La Laguna, Tenerife, Spain \\ ${ }^{3}$ Departamento de Astrofísica, Universidad de La Laguna, E-38205 La Laguna, Tenerife, Spain
}

\begin{abstract}
Barlenses are morphological features present in barred galaxies with morphological types between S0 and Sb. Recently, they have been suggested to be the more face-on counterparts of the boxy/peanut bulges generally observed in edge-on galaxies. In this work we test this idea by looking at the orientations and colors of barlenses, and by comparing them with those of the bars and disks in the same galaxies. We find that barlenses have colors which are similar to those of bars, and also similar to those of elliptical galaxies.
\end{abstract}

Keywords. Galaxies: bulges, Galaxies: colors

\section{Introduction}

Barlens (bl; Laurikainen et al. 2011) is a lens-like structure embedded in the bar of galaxies with morphological types from S0 to Sb. Its size is typically half the size of the bar. In simulations, the boxy/peanut $(\mathrm{B} / \mathrm{P})$ bulges seen in the edge-on view appear as barlenses in low inclination galaxies (Athanassoula et al. 2015). Laurikainen et al. (2014) suggested that the galaxies showing $\mathrm{B} / \mathrm{P} / \mathrm{X}$-shaped bulges and those that host barlenses form a single population in terms of stellar mass and axis ratio. They also show that the surface brightness profiles along the minor and major axes of the bar of a simulated $\mathrm{B} / \mathrm{P}$ bulge, that resembles a bl in face-on view, are very similar to those of an observed barlens galaxy. The above evidence links barlenses to bars and $\mathrm{B} / \mathrm{P}$ bulges. If this hypothesis is true, we would expect barlenses to behave like vertically thick structures in isophotal analysis, and their colors to be similar to those of bars.

\section{Sample and methods}

Our sample consists of 79 barlens galaxies from two infrared surveys: the Spitzer Survey of Stellar Structure in Galaxies ( $\mathrm{S}^{4} \mathrm{G}$; Sheth et al. 2010) and the Near-Infrared S0 Galaxy Survey (NIRS0S; Laurikainen et al. 2011). The optical data consist of mosaics in u, g, r, i, z, SDSS bands from Knapen et al. (2014), for 45 of the barlens galaxies in our sample. The methods we adopted were the following:

(1) We measured the semimajor axis length $\left(\mathrm{r}_{\mathrm{bl}}\right)$, axis ratio $\left(\mathrm{b} / \mathrm{a}_{\mathrm{bl}}\right)$ and orientation $\left(\mathrm{PA}_{\mathrm{bl}}\right)$ of a barlens in the sky-plane, using the infrared images, by fitting an ellipse to points marked on a given isophotal level that describes the bl.

(2) In order to obtain the color indices, we selected regions for the different structures on the optical images of the barlens galaxies. Two approaches were used, manual and automatic (see Fig. 1). For the automatic approach we used the parameters obtained for 

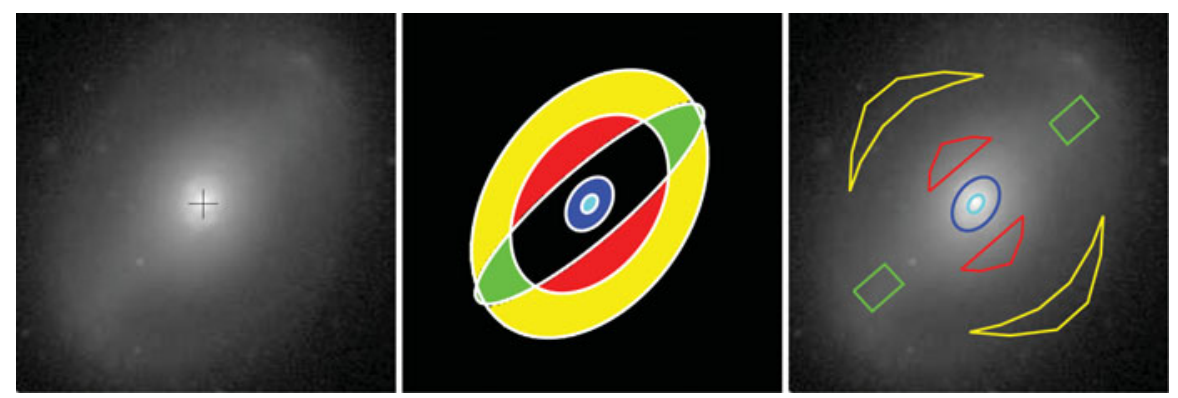

Figure 1. Example of the regions selected to obtain the colors in our sample. Shown are the barlens galaxy NGC 4245 in r-band (left), and the two approaches: automatic (center) and manual (right). Nuclear regions are the same in both approaches.

the barlens $\left(\mathrm{r}_{\mathrm{bl}}, \mathrm{b} / \mathrm{a}_{\mathrm{bl}}\right.$, and $\left.\mathrm{PA}_{\mathrm{bl}}\right)$ in order to define the regions of interest (see Fig. 1, middle panel). For the nucleus, NUC1: cyan region, and NUC2: blue + cyan regions (NUC2 is larger than NUC1 in order to include any nuclear ring); for the barlens, BL: red regions; and for the DISK: yellow regions. For the BAR (i.e. the thin bar): green regions, the parameters (semimajor axis length, axis ratio and position angle) are taken from Laurikainen et al. (2011) or Herrera-Endoqui et al. (2015) for NIRS0S and $\mathrm{S}^{4} \mathrm{G}$ galaxies, respectively. The fluxes in the optical bands were extracted from these regions, converted into magnitudes, and corrected for Galactic extinction. The color indices obtained with both approaches are very similar and only those obtained using the automatic manner are shown in Fig. 2 (right).

\section{Barlenses are vertically thick structures}

It was shown by Erwin \& Debattista (2013) that B/P bulges can be identified by their isophotal behavior even in moderately inclined galaxies due to their vertical thickness with respect to the thin bars. According to the simulations of Bettoni \& Galletta (1994) and Athanassoula \& Beaton (2006), when the B/P bulge is seen not edge-on, it will have a different orientation than the bar, due to an inclination effect. In fact, it will appear oriented closer to the line of nodes than the thin bar. The same behavior is observed for barlenses in Fig. 2 (left). When the inclination is low, the bl is mainly oriented parallel to the bar, however, as the inclination increases, the orientation of the bl gets closer to the line of nodes. B/P bulges would appear in this plot as barlenses at intermediate galaxy inclinations.

\section{Conclusions}

We compared the optical colors of barlenses and thin bars, using different color indices. We use indices sensitive to metallicity $(u-g)$ and old stars $(r-i$ and $i-z)$. In Fig. 2 (right) we show histograms of the difference in color between the different structures as bars, nuclei and disks with respect to the colors of the barlens. The general behavior is that nuclei are redder and disks are bluer than barlenses. However, thin bars have similar colors as barlenses as seen by their almost zero deviations in their color differences, suggesting similar stellar population content. The obtained colors and isophotal analysis are consistent with the idea that barlenses are vertically thick and form part of the bar.

We have shown that the optical colors of barlenses are very similar to those of the thin bar (see Fig. 2, right). Also their orientation with respect to the bar and the line of nodes 

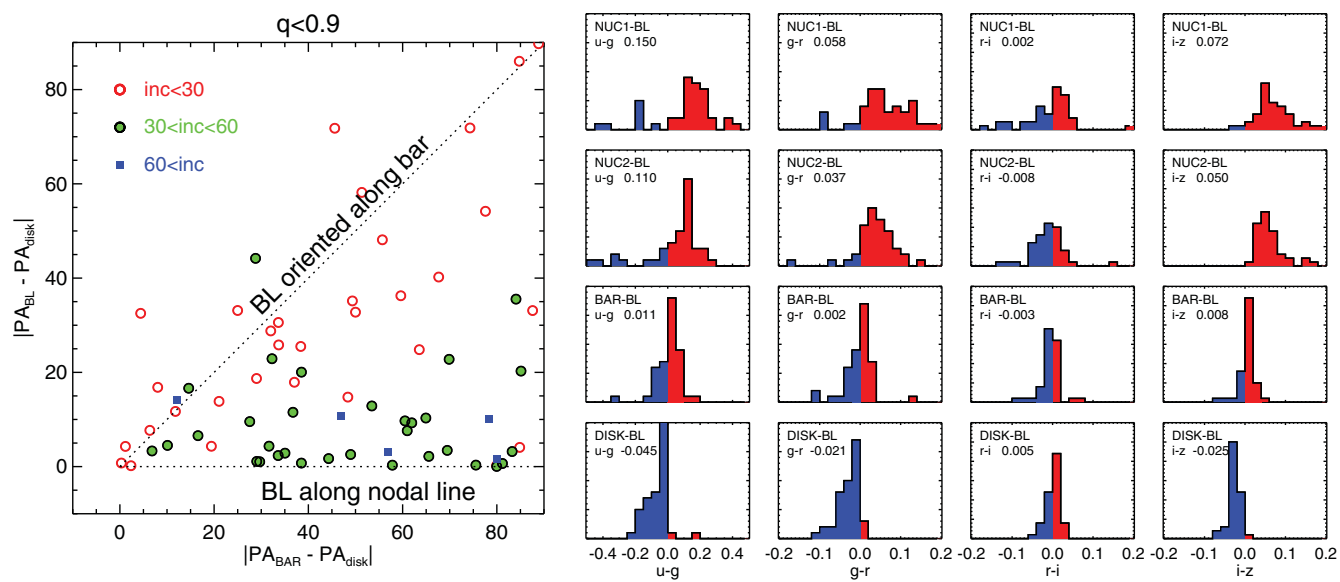

Figure 2. Left: orientation of barlenses with respect to the bar and the line of nodes in bins of galaxy inclination (the data for the orientation of the line of nodes and galaxy inclination are taken from Salo et al. 2015 or Laurikainen et al. 2011 for $\mathrm{S}^{4} \mathrm{G}$ and NIRS0S galaxies, respectively). Right: color deviations of the structures with respect to the barlenses. The numbers represent the median value of the deviations. Deviations to bluer or redder colors are color coded.

at different galaxy inclinations is consistent with the idea that that they are vertically thick structures of bars.

\section{Acknowledgements}

We acknowledge support to the DAGAL network from the People Programme (Marie Curie Actions) of the European Union's Seventh Framework Programme FP7/2007-2013/ under REA grant agreement PITN-GA-2011-289313. JHK thanks the support from the Spanish Ministry of Economy and Competitiveness (MINECO) under grant number AYA2013-41243-P, the Astrophysics Research Institute of Liverpool John Moores University for their hospitality, and the Spanish Ministry of Education, Culture and Sports for financial support of his visit there, through grant number PR2015-00512.

\section{References}

Athanassoula, E. \& Beaton, R. L. 2006, MNRAS, 370, 1499

Athanassoula, E., Laurikainen, E., Salo, H., \& Bosma, A. 2015, MNRAS, 454, 3843

Bettoni, D. \& Galletta, G. 1994, A\&A, 281, 1

Erwin, P. \& Debattista, V. P. 2013, MNRAS, 431, 3060

Herrera-Endoqui, M., Díaz-García, S., Laurikainen, E., \& Salo, H. 2015, A\&\&A, 582, A86

Knapen, J. H., Erroz-Ferrer, S., Roa, J., et al. 2014, A\&A, 569, A91

Laurikainen, E., Salo, H., Buta, R., \& Knapen, J. H. 2011, MNRAS, 418, 1452

Laurikainen, E., Salo, H., Athanassoula, E., Bosma, A., \& Herrera-Endoqui, M. 2014, MNRAS (Letters) 444, L80

Salo, H., Laurikainen, E., Laine, J., et al. 2015, ApJS, 219, 4

Sheth, K., Regan, M., Hinz, J. L., et al. 2010, PASP, 122, 1397 